\title{
Efeitos benéficos do exercício físico no tratamento da depressão: uma revisão integrativa
}

\author{
Beneficial effects of physical exercise in the treatment of depression: an integrative review \\ Efectos beneficiosos del ejercicio físico en el tratamiento de la depresión: una revisión integradora
}

Recebido: 05/01/2022 | Revisado: 09/01/2022 | Aceito: 14/01/2022 | Publicado: 16/01/2022

\author{
Rodrigo Yan Candido da Silva \\ ORCID: https://orcid.org/0000-0001-5465-6812 \\ Centro Universitário Mário Pontes Jucá, Brasil \\ E-mail: rodrigoycs@hotmail.com \\ Ailton Mota do Nascimento Galvão \\ ORCID: https://orcid.org/0000-0002-9268-6921 \\ Centro Universitário Mário Pontes Jucá, Brasil \\ E-mail: ailton_fisioterapeuta@ hotmail.com \\ Elenildo Aquino dos Santos \\ ORCID: https://orcid.org/0000-0001-8404-9001 \\ Centro Universitário Mário Pontes Jucá, Brasil \\ E-mail: elenildoa@hotmail.com \\ Ana Clara dos Santos Barbosa \\ ORCID: https://orcid.org/0000-0003-2583-9999 \\ Centro Universitário Mário Pontes Jucá, Brasil \\ E-mail: bibiclara@ hotmail.com.br
}

\begin{abstract}
Resumo
Introdução: Nos dias atuais, a depressão é uma das causas mais incapacitantes mundialmente, podendo acometer todas as pessoas, afetando diretamente em seu cotidiano como: trabalho, relações sociais, família e outros (Silva et al., 2019). O exercício físico é importante aliado na prevenção e tratamento da depressão, promovendo o bem-estar para o paciente, visando a mudança de hábitos no dia-a-dia e uma melhor qualidade de vida. (Silva, Vieira, \& Brito, 2019) Objetivo: conscientização sobre a importância dos exercícios físicos na prevenção e tratamento da depressão. Metodologia: Revisão integrativa da literatura, com busca nas bases de dados SciELO, Lilacs e PubMed, colhendo trabalhos publicados em português e inglês nos últimos 5 anos (2017-2021). Resultados: Foram encontrados 143 artigos conforme os critérios de inclusão e exclusão, sendo utilizados para a discussão apenas 8. Discussão: A grande maioria dos artigos apontou que o exercício físico associado a terapia farmacológica tem efeitos benéficos, embora necessitem de pesquisas com menos riscos de viés. Conclusão: $O$ exercício físico deve ser implementado no tratamento de pessoas diagnosticadas com depressão, sendo acompanhado por um profissional capacitado, tem grandes chances de obter resultados satisfatórios.
\end{abstract}

Palavras-chave: Exercício físico; Depressão; Tratamento.

\begin{abstract}
Introduction: Nowadays, depression is one of the most disabling causes worldwide, affecting all people, directly affecting their daily lives such as: work, social relationships, family and others. (Silva et al., 2019). Physical exercise is an important ally in the prevention and treatment of depression, promoting well-being for the patient, aiming at changing daily habits and improving quality of life. (Silva, Vieira, \& Brito, 2019) Objective: awareness of the importance of physical exercise in the prevention and treatment of depression. Methodology: Integrative literature review, with a search in SciELO, Lilacs and PubMed databases, collecting works published in Portuguese and English in the last 5 years (2017-2021). Results: 143 articles were found according to the inclusion and exclusion criteria, being used for the discussion only 8. Discussion: The vast majority of articles indicated that physical exercise associated with pharmacological therapy has beneficial effects, although they need research with less risk of bias. Conclusion: Physical exercise should be implemented in the treatment of people diagnosed with depression, being accompanied by a trained professional, it has great chances of obtaining satisfactory results.
\end{abstract}

Keywords: Physical exercise; Depression; Treatment.

\section{Resumen}

Introducción: En la actualidad, la depresión es una de las causas más discapacitantes a nivel mundial, afectando a todos, afectando directamente su vida cotidiana como: trabajo, relaciones sociales, familia y otros (Silva et al., 2019). El ejercicio físico es un aliado importante en la prevención y el tratamiento de la depresión, favoreciendo el bienestar del paciente, con el objetivo de cambiar los hábitos diarios y mejorar la calidad de vida. (Silva, Vieira, \& Brito, 2019) 
Objetivo: concienciar sobre la importancia del ejercicio físico en la prevención y tratamiento de la depresión. Metodología: Revisión integrativa de la literatura, con búsqueda en bases de datos SciELO, Lilacs y PubMed, recogiendo trabajos publicados en portugués e inglés en los últimos 5 años (2017-2021). Resultados: Se encontraron 143 artículos según los criterios de inclusión y exclusión, siendo utilizados solo para la discusión 8. Discusión: La gran mayoría de los artículos indicaron que el ejercicio físico asociado a la terapia farmacológica tiene efectos beneficiosos, aunque necesitan investigación con menor riesgo de sesgo. Conclusión: El ejercicio físico debe ser implementado en el tratamiento de personas diagnosticadas con depresión, estando acompañado de un profesional capacitado, tiene grandes posibilidades de obtener resultados satisfactorios.

Palabras clave: Ejercicio físico; Depresión; Tratamiento.

\section{Introdução}

Atualmente a depressão é vista como uma das doenças mais incapacitantes a nível mundial, podendo acometer pessoas de todas as idades, gêneros e classes, interferindo em todas as suas esferas sociais, sejam elas relações interpessoais, trabalho, aceitação própria, família e afins (Silva, Vieira, \& Brito, 2019). Possíveis fatores que estão associados ao desenvolvimento da depressão, são: genéticos, hormonais, problemas emocionais, crenças, conflitos sociais, uso de entorpecentes, entre outros. (Silva \& Santos, 2019)

Gastos com saúde mental, liderados por despesas com medicamentos aumentaram consideravelmente nas últimas décadas, evidenciando que as atuais abordagens não estão sendo suficientes para prevenir e tratar esses distúrbios, as gerações mais jovens aparentam ter um aumento de transtornos mentais como depressão e ansiedade. Para muitas pessoas com esse tipo de distúrbio mental a medicação não resulta em resultados benéficos a longo prazo, podendo desencadear efeitos colaterais como ganho de peso considerável, elevação do nível de glicose no sangue, perda da libido, e outros. Esses efeitos podem causar o abandono ao tratamento e impactar negativamente a vida do paciente. (Schuch \& Vancampfort, 2021)

O exercício físico é um grande aliado na prevenção e combate a depressão, devendo sempre ser sempre orientado por um profissional capacitado, tendo um excelente efeito terapêutico. A prática regular de exercícios físicos interfere positivamente em todos os nossos sistemas, seja ele respiratório, psíquico, cardíaco, osteomioarticular e outros. A terapia farmacológica é a mais aplicada no tratamento da depressão, porém, quando não possuem uma boa adesão do paciente os exercícios físicos são de suma importância para prevenção e tratamento. (Santiago, 2017)

De acordo com a Organização Mundial de Saúde (OMS) a depressão é um dos distúrbios mais importantes, ocupando o segundo lugar e ficando atrás apenas dos problemas cardiovasculares, por isso sua prevenção e tratamento é tão abordada atualmente, principalmente nos casos de remissão, embora o alívio sintomas seja um dos principais objetivos do tratamento, a manutenção da saúde desse indivíduo de modo geral é um dos desafios atuais em saúde física e mental. (Parami et al., 2021)

Muitos problemas psicológicos começam a ser desenvolvidos na adolescência, época em que passamos por diversas mudanças biopsicossociais, construindo a personalidade, moldando ideias e influências, tornando-se uma fase extremamente propícia ao aparecimento de transtornos depressivos e ansiedade. Algumas características da depressão são: irritabilidade, inconsistência emocional e alterações comportamentais, também podem aparecer cefaleias, fadiga física e dores abdominais, resultando num mal rendimento nas atividades de vida diárias e tendência ao uso de álcool e drogas. (Silva et al., 2019)

As chances de acometimento da depressão ao longo da vida são de $16 \%$, tendo um desfecho crônico, pessoas com transtornos mentais graves, incluindo a depressão, tem uma expectativa de vida cerca de 10 a 17,5 anos mais curta em comparação com as pessoas de modo geral. Entre esse público específico, pesquisadores atentam pelas elevadas taxas de mortalidade, causadas também pelos prejuízos adversos a saúde, como dieta inadequada, tabagismo, sedentarismo, uso de determinadas substâncias, entre outros. (Monteiro et al., 2021)

A fisioterapia é importante aliada na prevenção e tratamento da depressão, sendo competente para desempenhar técnicas que promovam bem-estar para o paciente, visando a mudança de hábitos no dia-a-dia e uma melhor qualidade de vida. (Silva et al., 2019). Exercícios físicos tendem a ser os mais indicados se praticados ao menos 3 vezes por semana, mostrando- 
se mais eficazes que treinos semanais únicos, sendo uma terapia mais em conta, torna-se uma boa escolha pelos resultados, atuando como regulador neural e físico. (Cordeiro, 2020)

Atualmente tem-se abordado o que realmente é necessário fazer para ter uma melhor qualidade de vida, em meio a correria e falta de tempo da vida moderna, as pessoas buscam cada vez mais por melhorias na saúde, tendo em vista que o capitalismo implementou novas formas de trabalho, vida social e relações interpessoais, exigindo maiores esforços para dar conta de todas as responsabilidades. Nesse meio, muitas vezes acabam surgindo os transtornos depressivos, influenciando negativamente no condicionamento físico, prejudicando a saúde mental, atingindo níveis sociais e emocionais. (Ferreira, 2017)

Sabemos que a depressão vem se multiplicando ao longo dos anos, chegando a ser considerada o "mal do século", sendo assim, é importante frisar que a grande maioria das pessoas podem ser beneficiadas pela prática de exercícios físicos, diminuindo os sintomas depressivos, transtornos de humor, diminuição de dores no corpo, aumentando a autoestima, qualidade de vida, entre outros; as melhorias podem ser vistas tanto a nível intrínseco quanto extrínseco. (Santiago, 2017)

Os exercícios físicos podem estar correlacionados a liberação de hormônios, como de conhecimento, também desempenham papel importante no humor e saúde mental. Os exercícios favorecem principalmente a liberação de endorfinas, que agem no cérebro reduzindo a percepção de dor, como sedativos e analgésicos, sendo assim, sua implementação no tratamento da depressão é de grande valia, pois, mostra-se muitos eficaz principalmente nos casos leves e moderados da depressão. (Shape \& Chahal, 2010)

A falta de exercícios é um fator que causa um impacto muito grande na saúde do indivíduo, seja ela física ou mental, muita das vezes começa com a diminuição da autoestima, do bem-estar, da satisfação e da socialização, acontece também o aumento do estresse, ansiedade e possivelmente a depressão; essas pessoas tem uma forte tendência a desenvolverem outras doenças, que com a prática do exercício físico poderiam ser controladas com mais facilidade, como o controle do peso corporal por exemplo. (Santos, 2019).

Diante de tal situação, os transtornos mentais passaram a ser estudados a partir de diversas abordagens visando uma melhor compreensão, afim de encontrar novas terapêuticas, entretanto, não é um assunto que despertou a curiosidade recentemente, estudiosos do passado já procuravam desvendar esses transtornos. Podem ser classificados como transtorno disruptivo da regulação do humor, transtorno depressivo maior, transtorno depressivo persistente, transtorno disfórico prémenstrual, transtorno depressivo induzido por substância e/ou medicamento, transtorno depressivo desenvolvido por outra condição médica, transtorno depressivo especificado e não especificado, tendo todos eles características em comum. (Balleoti, 2018).

Pessoas que são diagnosticadas ou tem sinais e sintomas característicos da depressão tendem a proporcionar maiores gastos para a saúde, incapacidade para atividades laborais e dificuldade de aceitação a terapias farmacológicas, comumente está associada a existência de patologias já instaladas, tornando-os mais vulneráveis aumentando o risco de acometimento pela mesma (Silva, Vieira, \& Brito, 2019). Este trabalho tem como objetivo a conscientização de indivíduos e profissionais sobre a importância dos exercícios físicos na prevenção e tratamento da depressão.

\section{Metodologia}

O presente artigo trata-se de uma revisão integrativa da literatura, foram usados os seguintes Descritores em Ciências da Saúde (DeCS): "physical exercise", "depression" e "treatment” com a combinação do operador booleano AND. As pesquisas foram feitas nas seguintes bases de dados: Scientific Eletronic Library Online (SciELO), Literatura Latino-americana e do Caribe em Ciências da saúde (LILACS) e United States National Library of Medicine (PubMed), a estratégia usada na busca bibliográfica e fluxograma com a demonstração da inclusão e exclusão dos estudos estão descritas na Figura 1. 
Os critérios de inclusão foram artigos em português e inglês, de acordo com o tema a ser abordado, publicados no período de 2017 a 2021 e disponíveis na integra gratuitamente. Os critérios de exclusão foram artigos que não se enquadraram no objetivo do estudo e não correspondiam aos critérios de inclusão determinados, também foram excluídos estudos que abordavam o tema associado a outras patologias, técnicas especificas para tratamento e distinção de gênero.

Os materiais obtidos mediante o cruzamento dos Descritores em Ciências da Saúde (DeCS) e o operador booleano $A N D$, apresentaram-se da seguinte maneira: ("physical exercise") AND (“depression") AND ("treatment”). Após a leitura dos títulos e resumos, foram selecionados os materiais que melhor se enquadraram no objetivo do estudo respeitando os critérios de inclusão e exclusão.

\subsection{Figuras e tabelas}

Figura 1. Fluxograma de demonstração das etapas para seleção dos estudos revisados.

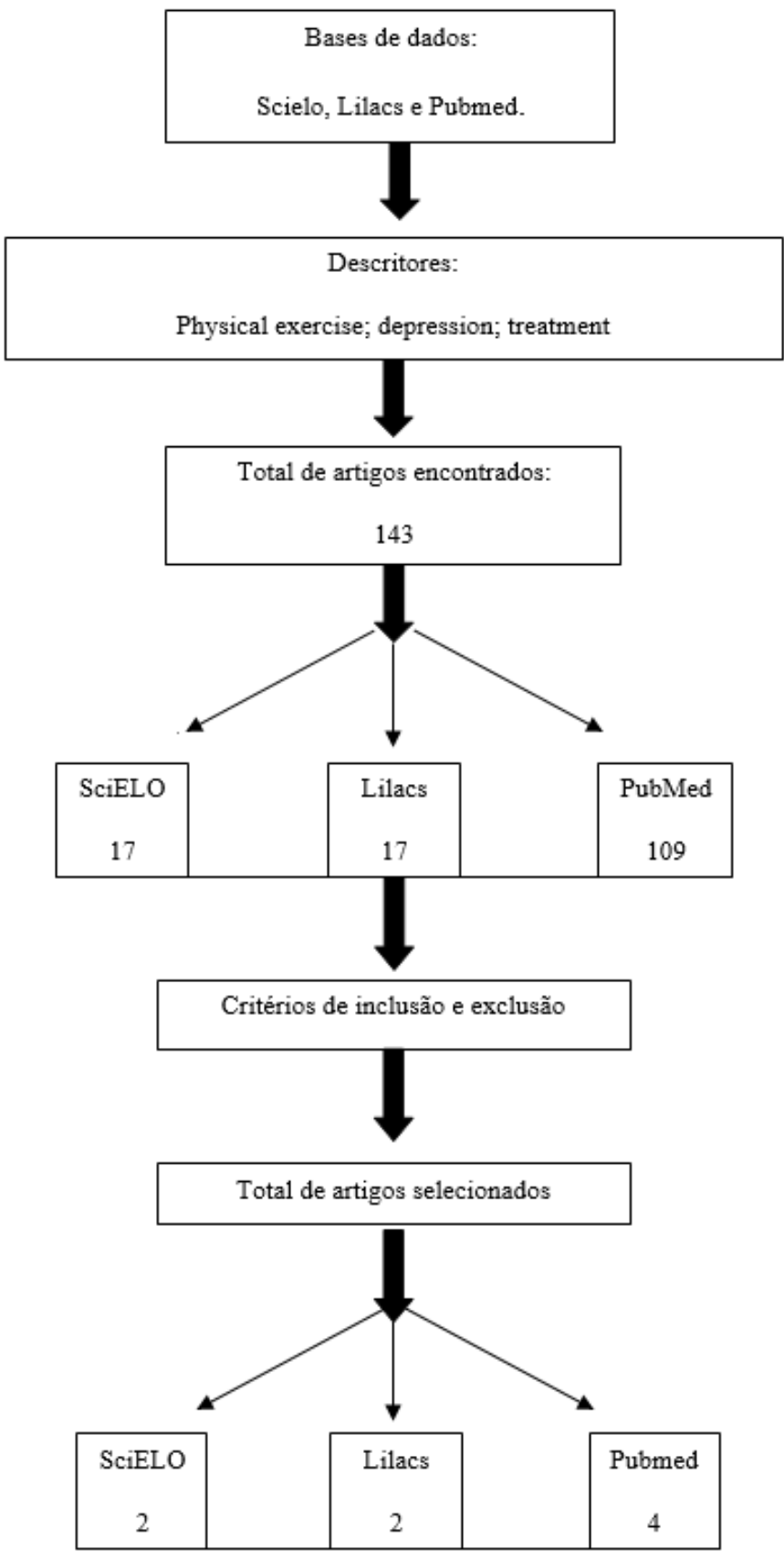

Fonte: Elaborado pelos autores. 


\section{Resultados e Discussão}

Durante a coleta de dados mediante os critérios de inclusão e exclusão, restaram apenas 8 estudos, sendo excluídos 135 estudos do total encontrado nas bases de dados. Dos materiais revisados, quatro foram encontrados na plataforma Pubmed, dois na plataforma SciElo e dois na plataforma Lilacs. Dentre os artigos, quatro foram publicados no ano de 2019, dois no ano de 2018 e dois no ano de 2017. A seguir, expresso na Tabela 1, dados apresentados nos trabalhos selecionados.

Tabela 1. Quadro sinóptico com estudos revisados conforme autor, ano, objetivo, método e resultado.

\begin{tabular}{|c|c|c|c|}
\hline AUTOR/ ANO & OBJETIVO & MÉTODOS & RESULTADOS \\
\hline Dang (2019) & $\begin{array}{l}\text { Observar as características } \\
\text { dos transtornos depressivos } \\
\text { examinando a influência do } \\
\text { exercício físico na } \\
\text { depressão. }\end{array}$ & $\begin{array}{l}\text { O artigo compara e analisa a relação } \\
\text { entre exercícios físicos, o nível de } \\
\text { depressão e autoestima corporal na } \\
\text { perspectiva da psicologia. }\end{array}$ & $\begin{array}{l}\text { Existem diferenças significativas na atitude } \\
\text { em relação ao exercício físico e o grau de } \\
\text { depressão nos três tipos de sujeitos do } \\
\text { estudo. O exercício físico é propício para o } \\
\text { alívio da depressão. }\end{array}$ \\
\hline $\begin{array}{l}\text { Gomes, A., Ramos, } \\
\text { S., Ferreira, A. R., } \\
\text { Montalvão, J., } \\
\text { Ribeiro, I., \& Lima, } \\
\text { F. (2019) }\end{array}$ & $\begin{array}{l}\text { Conhecer a efetividade do } \\
\text { exercício físico no } \\
\text { tratamento da perturbação } \\
\text { depressiva. }\end{array}$ & $\begin{array}{l}\text { Revisões sistemáticas de eficácia que } \\
\text { utilizam a estratégia PICO e as } \\
\text { recomendações PRISMA dirigidas } \\
\text { pelo Joanna Briggs Institute e } \\
\text { partindo da questão, qual a } \\
\text { efetividade do exercício físico no } \\
\text { tratamento da depressão? }\end{array}$ & $\begin{array}{l}\text { Identificaram } 310 \text { resultados. Fizeram cinco } \\
\text { documentos, quatro resultados da pesquisa } \\
\text { efetuada nas plataformas de acesso e } \\
\text { resultado da consulta de referências } \\
\text { bibliográficas. A seleção foi feita após leitura } \\
\text { do título; eliminação de duplicados; leitura de } \\
\text { resumos e textos integrais. }\end{array}$ \\
\hline $\begin{array}{l}\text { Schuch \& Stubbs, } \\
2019\end{array}$ & $\begin{array}{l}\text { Fornecer uma visão geral } \\
\text { atualizada do uso do } \\
\text { exercício para a prevenção e } \\
\text { tratamento da depressão. }\end{array}$ & Revisão sistemática da literatura. & $\begin{array}{l}\text { A literaturas recentes demonstraram que } \\
\text { níveis mais elevados de atividade física e } \\
\text { exercícios conferem efeitos protetores na } \\
\text { depressão incidente. }\end{array}$ \\
\hline $\begin{array}{l}\text { Murri, M. B. et al. } \\
\text { (2019) }\end{array}$ & $\begin{array}{l}\begin{array}{l}\text { Revisar a } \\
\text { abordando } \\
\text { equívocos }\end{array} \\
\text { mencionados nos trabalhos } \\
\text { e fornecer recomendações } \\
\text { práticas para prescrição de } \\
\text { exercícios para pessoas com } \\
\text { depressão. }\end{array}$ & Revisão narrativa da literatura. & $\begin{array}{l}\text { O exercício físico tem se mostrado um } \\
\text { tratamento eficaz para depressão maior, em } \\
\text { vários ensaios randomizados controlados } \\
\text { foram comparando-o a uma ampla gama de } \\
\text { outros tratamentos, incluindo cuidados } \\
\text { habituais, intervenções psicológicas, e } \\
\text { medicamentos antidepressivos. }\end{array}$ \\
\hline $\begin{array}{l}\text { Beserra, A. H. N., } \\
\text { Kameda, } \\
\text { Deslandes, A. } \\
\text { Schuch, F. } \\
\text { Laks, J., \& Moraes, } \\
\text { H. S. (2018) }\end{array}$ & $\begin{array}{l}\text { Revisar as evidências de } \\
\text { alterações de cortisol após o } \\
\text { exercício de pessoas com } \\
\text { depressão. }\end{array}$ & $\begin{array}{l}\text { Procedeu-se a meta-análise com o } \\
\text { cálculo do tamanho do efeito da } \\
\text { diferença média padronizada. Foi } \\
\text { utilizado o software Stata v.11.0. }\end{array}$ & $\begin{array}{l}\text { Houve redução dos níveis de cortisol no } \\
\text { grupo que realizou os exercícios. }\end{array}$ \\
\hline $\begin{array}{lr}\text { Carneiro, L. } & \text { F., } \\
\text { Mota, M. } & \text { P., } \\
\text { Schuch, } & \text { F., } \\
\text { Deslandes, A., } & \& \\
\text { Vasconcelos, } & \text { R. } \\
\text { J. } \\
(2017)\end{array}$ & $\begin{array}{l}\text { Reenfatizar a importância } \\
\text { de preencher essa lacuna e } \\
\text { integrar o exercício à } \\
\text { prática clínica como um } \\
\text { componente essencial do } \\
\text { tratamento da depressão. }\end{array}$ & $\begin{array}{l}\text { Dois RCTs realizados em Portugal } \\
\text { investigaram o efeito do exercício } \\
\text { como um tratamento para depressão. }\end{array}$ & $\begin{array}{l}\text { Em uma amostra de resistente ao tratamento } \\
\text { pacientes com transtorno depressivo maior, } \\
\text { descobriram que nenhum dos sujeitos no } \\
\text { grupo de controle (farmacoterapia sozinha) } \\
\text { exibiu resposta clínica ou remissão, enquanto } \\
\text { o grupo de intervenção (exercício + } \\
\text { farmacoterapia) apresentou uma taxa de } \\
\text { resposta clínica de } 21 \% \text { e taxa de remissão de } \\
26 \% \text {. Nosso estudo mostrou que o exercício é } \\
\text { um coadjuvante eficaz para medicamentos } \\
\text { antidepressivos para adultos com depressão, } \\
\text { diminuindo seus sintomas e aumento da } \\
\text { aptidão física. }\end{array}$ \\
\hline
\end{tabular}




\begin{tabular}{|c|c|c|c|}
\hline $\begin{array}{l}\text { Toups, M., } \\
\text { Carmody, T., } \\
\text { Greer, T., Rethorst, } \\
\text { C., Grannemann, } \\
\text { B., \& Trivedi, M. } \\
\text { H. (2019) }\end{array}$ & $\begin{array}{l}\text { Avaliar o efeito do } \\
\text { exercício na valência } \\
\text { positiva nos sintomas de } \\
\text { Transtorno } \\
\text { Maior (MDD). }\end{array}$ & $\begin{array}{l}\text { Indivíduos em tratamento com } \\
\text { Aumento de Exercícios para } \\
\text { Depressão (TREAD) o estudo } \\
\text { completou SHAPS e MEI auto } \\
\text { relatados durante } 12 \text { semanas de } \\
\text { exercício para depressão. Avaliamos } \\
\text { os efeitos do exercício nas pontuações } \\
\text { SHAPS e MEI, e se as mudanças } \\
\text { foram relacionadas à gravidade geral } \\
\text { de MDD medida com o Quick } \\
\text { Inventory of Depression } \\
\text { Sintomatologia (QIDS). }\end{array}$ & $\begin{array}{l}\text { Shaps e pontuações MEI significativamente } \\
\text { melhorada com o exercício. Mudança de } \\
\text { pontuação MEI teve maior efeito e maior com } \\
\text { a mudança no escore QIDS. MEI também } \\
\text { mostrou significante correlação entre efeitos } \\
\text { moderadores e mediadores do exercício em } \\
\text { TDM. }\end{array}$ \\
\hline $\begin{array}{l}\text { Krogh, J., Hjorth } \varnothing, \\
\text { C., Speyer, H., } \\
\text { Gluud, C., \& } \\
\text { Nordentoft, } \\
\text { (2017) }\end{array}$ & $\begin{array}{l}\text { Avaliar os benefícios e } \\
\text { malefícios do exercício } \\
\text { físico em pacientes com } \\
\text { depressão. }\end{array}$ & $\begin{array}{l}\text { Revisão sistemática com meta-análise } \\
\text { e ensaio de análise sequencial. }\end{array}$ & $\begin{array}{l}\text { Trinta e cinco ensaios envolvendo } 2.498 \\
\text { participantes foram incluídos. O efeito do } \\
\text { exercício versus controle da gravidade na } \\
\text { depressão foi }-0,66 \text { média padronizada com } \\
\text { diferença (SMD) (IC } 95 \%-0,86 \text { a }-0,46 ; p \\
<0,001 \text {; classificação de recomendações, } \\
\text { avaliação, desenvolvimento e avaliação } \\
\text { (GRADE): qualidade muito baixa). } \\
\text { Restringindo esta análise para os quatro } \\
\text { ensaios que pareciam menos afetados por } \\
\text { viés, o efeito desapareceu em baixa } \\
\text { qualidade. Exercício diminuiu o risco relativo } \\
\text { de não remissão com qualidade muito baixa. } \\
\text { Análise sequencial de ensaio aleatório com } \\
\text { erro foi excluída quando todas as tentativas } \\
\text { foram analisadas, mas não foi focado em } \\
\text { ensaios menos afetados de viés. Análises de } \\
\text { subgrupos encontradas, o tamanho do ensaio } \\
\text { e a duração da intervenção foram } \\
\text { inversamente associados ao tamanho do } \\
\text { efeito para a gravidade da depressão e falta de } \\
\text { remissão. Não houve efeito significativo de } \\
\text { exercício em resultados secundários. }\end{array}$ \\
\hline
\end{tabular}

Fonte: Elaborado pelos autores.

Sabemos que a depressão avançou consideravelmente nos últimos anos, sendo recomendado a implementação de exercícios físicos no tratamento dos sintomas, melhorando inclusivamente capacidades aeróbicas e qualidade de vida conforme citado na literatura. Infelizmente ainda não é tão recomendado como deveria, pois, alguns médicos alegam não terem bases sólidas referentes aos benefícios, mas nesses estudos veremos que sim, existe comprovação da sua eficácia, acarretando então no desenvolvimento de diretrizes brasileiras para o tratamento. (Carneiro et al., 2018)

De acordo com Beserra et al., (2019) a fisiopatologia da depressão está relacionada com alterações neurológicas, acarretando num funcionamento imperfeito do eixo hipotálamo-pituitária-adrenal (HPA) e consequentemente o aumento ou diminuição dos níveis de cortisol, favorecendo o aparecimento dos sintomas da depressão. Concluiu-se que exercícios cinco vezes por semana trazem efeitos mais benéficos para regulação dos níveis de cortisol e controle da remissão dos sintomas da depressão.

Entretanto, não foi possível ter plena confiança nos efeitos benéficos causados pelo exercício físico sobre a depressão, expondo que as intervenções produzem poucos ou negligenciáveis efeitos antidepressivos visíveis, sem detalhar o quanto dos efeitos são causados por viés ou pelo próprio exercício físico. Nesse estudo, não foi avaliado possíveis efeitos benéficos em 
outros sistemas, como o cardiovascular por exemplo, sendo possivelmente analisados, tem grandes chances de constatarem benefícios sobre esses pacientes diagnosticados com depressão. (Krogh et al., 2017)

Murri et al., (2019) afirma que pacientes com depressão dificilmente recebem tratamento adequado e quando recebem tem grandes chances de permanecerem deprimidos ou recaírem após o tratamento. Um meio para tornar o tratamento mais efetivo é a implementação dos exercícios físicos, que por muitas vezes não é recomendado, embora tenham evidencias que o exercício oferece vários benefícios físicos, neutralizando mecanismos que aumentam o risco de mortalidade na depressão, sendo prescrito e aplicado corretamente pode ser tão eficaz quanto outros.

Segundo a afirmação de Toups et al., (2017) constatou-se que no decorrer do tratamento com exercício físico houve uma mudança significativa nos sintomas de valência positiva, anedonia, falta de motivação e energia, tendo resultados relevantes comprovados tanto clinica quanto cientificamente, evidenciou também que a anedonia, motivação e energia são independentes da depressão. Em geral os resultados mostram-se positivos no combate a depressão e seus sintomas devido ao efeito antidepressivo proporcionado pela prática de exercícios físicos.

Sabe-se que o grau de acometimento da depressão é maior nas mulheres que nos homens, porém, não chega a ser uma diferença significativa. Devido ao alto custo da psicoterapia e terapias antidepressiva o exercício físico torna-se um grande aliado pela fácil implementação, baixo custo e os bons resultados no tratamento e controle da depressão. Foi observado também resultados melhores nos indivíduos que praticaram atividades físicas mais vezes por semana. (Dang, 2019)

Segundo Schuch e Stubbs (2019) nos últimos quarenta anos uma gama de testes foi realizada para avaliar os efeitos do exercício físico no tratamento da depressão, com resultados obtidos em várias meta-análises, foi comprovado que os resultados positivos são muitos grandes relacionado aos efeitos antidepressivos, porém, a implementação dos exercícios no cotidiano de alguém não é uma tarefa das mais fáceis, principalmente se essa pessoa for portadora de depressão, tendo por muitas vezes uma alta taxa de evasão, com probabilidade de abandono maior que para outras populações clínicas.

A prática de exercício físico associado a terapia farmacológica tem mostrado um bom resultado no tratamento e controle dos sintomas apresentados na depressão, inclusive no que diz respeito a efeitos secundários, ainda que exista uma escassez de resultados mediante práticas randomizadas. Todos os estudos apontam que a regularidade da prática tem um efeito mais satisfatório e duradouro, exercícios com uma intensidade maior apresentaram um maior índice de benefícios e também uma maior taxa de abandono, vale a pena avaliar bem e escolher o tratamento adequado para resultados mais benéficos. (Gomes et al., 2019)

Os pontos positivos do uso do exercício transcendem os benefícios físicos e adentram nos mentais, possuindo vários guias de prática clínica emitidos pelo Instituto Nacional do Reino Unido por Health and Clinical Excellence (NICE), Associação Americana de Psiquiatria (APA) e Royal Australian e o Colégio de Psiquiatras da Nova Zelândia (RANZCP). Apesar das diferenças entre os mesmos, instigam que o exercício físico é importante e deve ser usado como opção para tratamento e controle dos sintomas da depressão. (Carneiro et al., 2018)

A depressão inicialmente é tratada por médicos de atenção primaria, psicólogos e psiquiatras, com base nos resultados sobre os efeitos que o exercício físico pode proporcionar as praticantes que são diagnosticados com depressão, outros profissionais como fisioterapeutas e educadores físicos devem ser incrementados para obterem um melhor resultado, principalmente no início, quando os pacientes apresentarem queixas de depressão, tornando o acompanhamento por uma equipe multidisciplinar. (Murri et al., 2019)

Foi possível observar bons prognósticos relacionados a fatores biológicos, clínicos, psicológicos e sociais, a interação entre dois ou mais fatores corrobora a uma maior taxa de resposta ao tratamento, porém, precisamos estar cientes que a prática não será eficaz para todos os pacientes e é preciso trabalhar com essas hipóteses para não comprometer o tratamento. Alguns 
métodos podem ser adotados para evitar o abandono do paciente a pratica do exercício físico, são eles a motivação autônoma, dosagem correta de exercício e suporte social. (Schuch \& Stubbs, 2019)

Alguns autores falam que os efeitos psicológicos do exercício em sua grande maioria estão atrelados aos efeitos psicossociais "placebos" ou "não específicos", como o suporte e atenção da equipe durante o tratamento, colocando o exercício físico como alternativa, não reconhecendo de fato sua importância para somar ao tratamento. Outra crença que acaba por vezes atrapalhando o tratamento é que o exercício físico melhora apenas o sono e alterações de apetite, entretanto, resultados comprovam que é eficaz também para o humor deprimido, anedonia e ideação suicida. (Murri et al., 2019)

\section{Considerações Finais}

Ao longo deste trabalho buscamos refletir sobre os benefícios que os pacientes diagnosticados com depressão podem ter se aderirem a prática de exercícios físicos, vários estudos apontam que o exercício é fundamental para um prognóstico positivo, tendo uma melhor adesão ao tratamento por também tratar-se de uma terapia de baixo custo e com poucos ou sem efeitos colaterais, certificando que o exercício deve ser prescrito e acompanhado por um profissional capacitado. Afim de sanar possíveis dúvidas relacionadas a implementação do exercício físico no tratamento da depressão e seus principais efeitos, faz-se necessário o desenvolvimento de mais ensaios clínicos randomizados para avaliar com menos riscos de viés os benefícios da prática de exercícios físicos no tratamento da depressão. Conclui-se que o exercício físico é fundamental para indivíduos com depressão e que a prática orientada pode trazer de volta o prazer em desempenhar atividades que antes já não faziam, devolvendo a esperança e a expectativa, enxergando a vida de uma forma totalmente diferente.

\section{Referências}

Baleotti, M. E. (2018, Outubro). Efeitos da atividade física na ansiedade e depressão: uma revisão bibliográfica. Fórum Científico Fema-Anais, Assis, SP, Brasil, XI. ISSN: 2446-4708. http://uniesp.edu.br/sites/_biblioteca/revistas/20180813144247.pdf

Belvederi Murri, M., Ekkekakis, P., Magagnoli, M., Zampogna, D., Cattedra, S., Capobianco, L., Serafini, G., Calcagno, P., Zanetidou, S., \& Amore, M. (2019). Physical Exercise in Major Depression: Reducing the Mortality Gap While Improving Clinical Outcomes. Frontiers in psychiatry, 9, 762. https://doi.org/10.3389/fpsyt.2018.00762

Beserra, A. H. N., Kameda, P., Deslandes, A. C., Schuch, F. B., Laks, J., \& Moraes, H. S. (2018). Can physical exercise modulate cortisol level in subjects with depression? A systematic review and meta-analysis. Trends in psychiatry and psychotherapy, 40(4), 360-368. https://doi.org/10.1590/2237-6089-20170155

Carneiro, L. F., Mota, M. P., Schuch, F., Deslandes, A., \& Vasconcelos, R. J. (2017). Portuguese and Brazilian guidelines for the treatment of depression: exercise as medicine. Revista brasileira de psiquiatria, 40(2), 210-211. https://doi.org/10.1590/1516-4446-2017-2272

Cordeiro, N. B. (2020). Exercício físico para crianças e adolescentes com depressão (Trabalho de conclusão de curso). Centro Universitário Fametro UNIFAMETRO, Fortaleza, CE, Brasil.

Dang, L. (2021). Physical Exercises in Releving the Current State of Depression. Revista Brasileira de Medicina do Esporte. 27(8), 776-778. https://www.scielo.br/j/rbme/a/TwgzWcDHCjLnxHBY7kr7DdB/

Ferreira, L. W. S. (2017). A importância do exercício físico no tratamento de pessoas portadoras de depressão o mal do século (Trabalho de conclusão de curso). Faculdade de Educação e Meio Ambiente - FAEMA, Ariquemes, RO, Brasil.

Gomes, A., Ramos, S., Ferreira, A. R., Montalvão, J., Ribeiro, I., \& Lima, F. (2019). A efetividade do exercício físico no tratamento da depressão. Revista Portuguesa de Enfermagem de Saúde Mental, (22), 58-64. https://doi.org/10.19131/rpesm.0264

Krogh, J., Hjorthø, C., Speyer, H., Gluud, C., \& Nordentoft, M. (2017). Exercise for patients with major depression: a systematic review with meta-analysis and trial sequential analysis. BMJ Open, 7(9). e 014820.

Monteiro, F. C., Schuch, F. B., Deslandes, A. C., Mosqueiro, B. P., Caldieraro, M. A., \& Fleck, M. (2021). Factors associated with adherence to sports and exercise among outpatients with major depressive disorder. Trends in psychiatry and psychotherapy, 43(2), 108-115. https://doi.org/10.47626/2237-60892019-0109

Parami, S., Tapak, L., Poorolajal, J., Moghimbeigi, A., \& Ghaleiha, A. (2021). Identifying factors associated with the hospital readmission rate among patients with major depressive disorder. BMC Psychiatry, 21, 542. https://doi.org/10.1186/s12888-021-03559-7

Rocha, L. H. M., Junior, R. F. B., Silva, W. P., Monteiro, E. R., \& Miranda, M. J. C. (2021). Os benefícios da prática de exercício físico no tratamento da depressão. Epitaya E-books, 1(8), 44-51. https://doi.org/10.47879/ed.ep.2021298p44 
Research, Society and Development, v. 11, n. 1, e58311125379, 2022

(CC BY 4.0) | ISSN 2525-3409 | DOI: http://dx.doi.org/10.33448/rsd-v11i1.25379

Santiago, J. O. (2017). Os benefícios do exercício físico no controle e prevenção da depressão e ansiedade (Trabalho de conclusão de curso). Faculdade de Educação e Meio Ambiente - FAEMA, Ariquemes, RO, Brasil.

Santos, M. C. B. (2019). O exercício físico como auxiliar no tratamento da depressão. Revista Brasileira de Fisiologia do Exercício, 18(2), 108-115. https://www.portalatlanticaeditora.com.br/index.php/revistafisiologia/article/view/3106

Schuch, F. B. \& Vancampfort, D. (2021). Physical activity, exercise, and mental disorders: it is time to move on. Tendências em psiquiatria e psicoterapia, 43(3), 177-184. https://doi.org/10.47626/2237-6089-2021-0237

Schuch, F. B., \& Stubbs, B. (2019). The Role of Exercise in Preventing and Treating Depression. Current sports medicine reports, 18(8), 299-304. https://doi.org/10.1249/JSR.0000000000000620

Shaphe, M. A., \& Chahal, A. (2020). Relation of Physical Activity with the Depression: A Short Review. Journal of lifestyle medicine, 10(1), 1-6. https://doi.org/10.15280/jlm.2020.10.1.1

Silva, L. C., \& Santos, N. M. L. (2019). Efeitos do exercício físico nos aspectos fisiológicos, psicológicos e sociais em pessoas com depressão. Revista Científico Eletrônica de Ciências Aplicas da FAIT, 14(2). http://www.fait.revista.inf.br/imagens_arquivos/arquivos_destaque/8rmACQy634bi0CY_2020-619-20-47-56.pdf

Silva, M. R, Paredes, P. F. M., \& Taddeo, P. S. (2019, Novembro). A dançaterapia como recurso terapêutico em adolescentes com depressão. Conexão Unifametro-Anais, Fortaleza, CE, Brasil, VII. ISSN: 2357-8645. https://www.doity.com.br/anais/conexaounifametro2019/trabalho/124009

Silva, R. F., Vieira, A. P. O., \& Brito, A. P. (2019). Efeitos positivos da fisioterapia na depressão através do exercício físico e hidroterapia. Scire Salutis, 9(1), 1-8. 2019. http://sustenere.co/index.php/sciresalutis/article/view/2755

Toups, M., Carmody, T., Greer, T., Rethorst, C., Grannemann, B., \& Trivedi, M. H. (2019). Exercise is an Effective Treatment for Positive Valence Symptoms in Major Depression. J Affect Disord, 209, 188-194. https://www.sciencedirect.com/science/article/abs/pii/S0165032716308618 